\title{
Sequential chemotherapy by CHOP and DHAP regimens followed by high-dose therapy with stem cell transplantation induces a high rate of complete response and improves event-free survival in mantle cell lymphoma: a prospective study
}

\author{
F Lefrère ${ }^{1}$, A Delmer ${ }^{2}$, F Suzan ${ }^{3}$, V Levy ${ }^{4}$, C Belanger ${ }^{1}$, M Djabarri ${ }^{5}$, B Arnulf ${ }^{1}$, G Damaj ${ }^{1}$, N Maillard ${ }^{1}$, V Ribrag ${ }^{6}$, \\ M Janvier ${ }^{7}$, C Sebban $^{8}$, R-O Casasnovas ${ }^{9}$, R Bouabdallah $^{10}, \mathrm{~F}$ Dreyfus ${ }^{5}$, V Verkarre $^{11}$, E Delabesse $^{12}, \mathrm{~F}^{\prime}$ Valensi $^{12}$, \\ E McIntyre $^{12}, \mathrm{~N}$ Brousse $^{11}$, B Varet $^{1}$ and O Hermine ${ }^{1}$ \\ ${ }^{1}$ Service d'Hématologie Adultes, Hôpital Necker, Paris, France; ${ }^{2}$ Service des Maladies du Sang Hôpital de l'Hotel-Dieu, Paris, France; \\ ${ }^{3}$ Service d'Onco-Hématologie, Hôpital André Mignot, Versailles, France; ${ }^{4}$ Département de Biostatistique Médicale, INSERM U444, Hôpital \\ Saint-Louis, Paris, France; ${ }^{5}$ Service d'Hématologie, Hôpital Cochin, Paris, France; ${ }^{6}$ Institut Gustave Roussy, Villejuif, France; ${ }^{7}$ Service \\ d'Oncologie, Centre René Huguenin, St-Cloud, France; ${ }^{8}$ Service d'Hématologie, Hôpital Edouard Herriot, Lyon, France; ${ }^{9}$ Service \\ d'Hématologie, Centre Hospitalier, Dijon, France; ${ }^{10}$ Service d'Hématologie, Institut Paoli-Calmette, Marseille, France; ${ }^{11}$ Service \\ d'Anatomopathologie, Hôpital Necker, Paris, France; and ${ }^{12}$ Laboratoire d'Hématologie, Hôpital Necker, Paris, France
}

\begin{abstract}
Mantle cell lymphoma (MCL) is a distinct clinico-pathological entity with a poor prognosis. We have conducted a prospective study in patients with MCL to evaluate a therapeutic strategy in which CHOP polychemotherapy was followed by DHAP if CHOP failed to induce complete remission. Responding patients then proceeded to an intensification therapy with autologous peripheral blood stem cell transplantation (APBSCT). Twenty-eight consecutive patients with newly diagnosed aggressive MCL were included. After four cycles of CHOP regimen, two complete responses (CR) were obtained $(7 \%)$ and $14(50 \%)$, five (18\%) and seven (25\%) patients achieved partial (PR), minor (MR) and no response, respectively (one patient died from septic complications during CHOP induction). The two patients in CR after CHOP underwent intensification with TBI, high-dose cyclophosphamide-etoposide and APBSCT. The other twentyfive patients received DHAP and in this group a response rate of $92 \%$ (21 CR (84\%), two PR (8\%)) was observed. Two patients had progressive disease. The twenty-three responding patients received high-dose therapy (TAM8 regimen: TBI-cytarabinemelphalan) followed by APBSCT. One of the two partial responding patients achieved CR after TAM8. After a median follow-up of 47.6 months (range, 14-70), seven patients have relapsed. Our data confirm that: (1) CHOP regimen induces a low CR rate in MCL; (2) CHOP plus DHAP appears to be much more efficient and allows a large proportion of patients to proceed to high-dose therapy in CR; (3) consolidation therapy including TBI and high-dose Arac-C followed by APBSCT may
\end{abstract} improve event-free survival.

Leukemia (2002) 16, 587-593. DOI: 10.1038/sj/leu/2402406

Keywords: mantle cell lymphoma; autograft; total body irradiation; CHOP; DHAP

\section{Introduction}

Mantle cell lymphoma (MCL) has been recently described as a distinct clinico-pathological entity characterized by a predominantly diffuse or nodular proliferation of atypical small B lymphoid cells effacing the nodal architecture. ${ }^{1,2}$ These cells are characterized by their immunophenotype $\left(\mathrm{CD}^{+}, \mathrm{CD}^{2} 3^{-}\right.$, $\left.\mathrm{CD}_{10}^{-}, \mathrm{SIgM}^{+}, \mathrm{SIgD}^{+}\right) . \mathrm{MCL}$ is frequently associated with the $t(11 ; 14)$ translocation, which results in the overexpression of cyclin D1 gene within the bcl-1 locus., ${ }^{3-7}$ Despite an initial relatively indolent clinical presentation, the prognosis of $\mathrm{MCL}$ remains poor with a median survival time of less than 3 years. ${ }^{3,8-11}$ Adverse prognostic factors such as poor performance status (PS), B symptoms, high LDH and $\beta 2$-microglobulin

Correspondence: O Hermine, Service d'Hématologie Adultes, Groupe Hospitalier Necker-Enfants Malades, 149-161 rue de Sévres, 75743 Paris Cedex 15, France; Fax: 33 (1) 44495280

Received 27 February 2001; accepted 16 November 2001 levels are observed in more than $50 \%$ of patients during the progression of the disease. However, although the international prognostic factor index may be applied to $\mathrm{MCL}$ patients, it does not allow clear discrimination of good and poor risk patients since both groups have a nearly comparable poor outcome. ${ }^{12,20}$

$\mathrm{MCL}$ is characterized by its refractoriness to most usual therapeutic strategies for lymphomas. Although $\mathrm{CHOP}$ or $\mathrm{CHOP}$-like regimens are considered as a reference therapy for non-Hodgkin's lymphoma (NHL), in MCL they have been reported to induce a low rate of complete response with a range between $10 \%$ and $50 \% .^{10,13}$ When we began our study (May 1995) no other regimen had demonstrated a better value in order to increase response rate, event-free survival and overall survival. As observed in other $\mathrm{NHL}$, a complete clinical response after initial therapy seems to be associated with a longer disease-free free survival (DFS), but in MCL the impact of CR on overall survival remains to be determined. Preliminary reports have suggested that high-dose therapy (HDT) especially with total body irradiation (TBI) and autologous peripheral blood stem cell transplantation (APBSCT) for patients with MCL increases response rates and seems to produce better DFS than conventional therapy. ${ }^{14}$ This treatment, however, has not been proved to cure any patients. Furthermore, APBSCT does not seem to increase response rate and median DFS in patients who are not in the first phase of their disease. ${ }^{14}$

Therefore, due to the poor prognosis of MCL treated with conventional chemotherapy and taking into account results of earlier studies, we initiated a prospective study based on the response to initial CHOP therapy with the aims of increasing response rates before APBSCT and increasing event-free and overall survival. Patients who achieved CR after four cycles of CHOP underwent TBI plus high-dose cyclophosphamide and etoposide (Cy-Eto) myeloablative regimen and APBSCT. Patients who did not enter CR after CHOP were treated by a salvage therapy with high-dose cytarabine regimen (DHAP) followed by TBI plus high-dose Ara-c plus melphalan

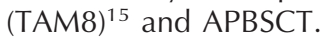

\section{Patients and methods}

\section{Patients eligibility}

After giving their informed consent, adult patients younger than 65 years with previously untreated aggressive $\mathrm{MCL}^{8}$ were enrolled in this prospective study. In accordance with Ann 
Arbor criteria, only patients with stages III and IV were included in the study. ${ }^{16}$ Patients with CNS involvement, leukemic phase were also eligible. After nodal biopsy, which was performed in all patients, aggressive MCL histological subtypes were included (nodular, diffuse or blastic). The diagnosis was based on a combination of morphologic, immunologic, cytogenetic and molecular criteria as previously described. ${ }^{2,7,8,14,17}$ Patients with mantle zone pattern, which is associated with a favorable course, were excluded from the study. ${ }^{18}$ All cases were reviewed by expert hematopathologists for confirmation of diagnosis.

Immunophenotypic studies were performed by flow cytometry or by in situ immunohistochemistry using antibodies, to the following cluster antigens, as previously described: CD3, CD5, CD19, CD10, CD20, CD23, CD43, FMC-7, immunoglobulin $\mathrm{M}(\lg \mathrm{M})$ and $\lg \mathrm{D}$. $^{7,17}$

Cytogenetic analysis, cyclin D1 mRNA expression and IgH/Bc11 translocation were performed when fresh or cryopreserved material was available as described earlier. ${ }^{6,7}$ Patients with any of the following were ineligible: cardiac ejection fraction less than 50\%, insufficient pulmonary function, severe renal or hepatic function impairment.

\section{Clinical evaluation}

Disease stage was evaluated in accordance with Ann Arbor criteria. ${ }^{16}$ The staging work-up included physical examination, blood cell count, liver function test, serum lactate deshydrogenase $(\mathrm{LDH})$ and $\beta 2$-microglobulin level determination, chest X-ray, computed tomography scans of the thorax, abdomen and pelvis, bone marrow aspirate and biopsy, gastrointestinal endoscopies and lumbar puncture for lymphomatous involvement.

\section{Protocol design}

The protocol design is shown in Figure 1. The initial chemotherapy consisted of four courses of CHOP regimen (cyclophosphamide $750 \mathrm{mg} / \mathrm{m}^{2}$ day 1, adriamycin $50 \mathrm{mg} / \mathrm{m}^{2}$ day 1 , vincristine $1.4 \mathrm{mg} / \mathrm{m}^{2}$ day 1 prednisone $40 \mathrm{mg} / \mathrm{m}^{2} /$ day days 1-5) with meningeal prophylaxis (methotrexate $10 \mathrm{mg}$, prednisone $40 \mathrm{mg}$ ) every 21 days. After the four CHOP courses, a complete evaluation, without bone marrow biopsy, was performed.

Patients in complete clinical response (clinCR) or in partial response (PR) were planned to have peripheral blood stem cell (PBSC) mobilization after cyclophosphamide $\left(4.5 \mathrm{~g} / \mathrm{m}^{2}\right)$ and etoposide (450 mg/m ${ }^{2}$ ) plus G-CSF (5 $\mu \mathrm{g} / \mathrm{kg} /$ day) administration (Cy-Eto). After PBSC collection, a new complete evaluation including bone marrow biopsy was performed. Patients in complete response (CR), including bone marrow biopsy, received two more courses of $\mathrm{CHOP}$ regimen followed by an intensive conditioning regimen with $\mathrm{TBI}$, cyclophosphamide (Cy, $60 \mathrm{mg} / \mathrm{kg} /$ day, days 1 and 2), etoposide (VP16, $300 \mathrm{mg} / \mathrm{m}^{2} /$ day, days $1-3$ ) plus APBSCT.

Patients in failure, in minor response or in progression after or during the CHOP regimen respectively and patients who did not reach CR after Cy-VP16 mobilization regimen were planned for a second line chemotherapy with 4 or 3 monthly courses of DHAP, respectively (cisplatin $100 \mathrm{mg} / \mathrm{m}^{2}$ day 1 , cytarabine 4 ( $2 \mathrm{~g} / 12 \mathrm{~h}) \mathrm{g} / \mathrm{m}^{2}$ day 2 , dexamethasone $40 \mathrm{mg} /$ day days $1-4)$. Patients who responded to DHAP were planned for PBSC harvested under G-CSF after the second course of

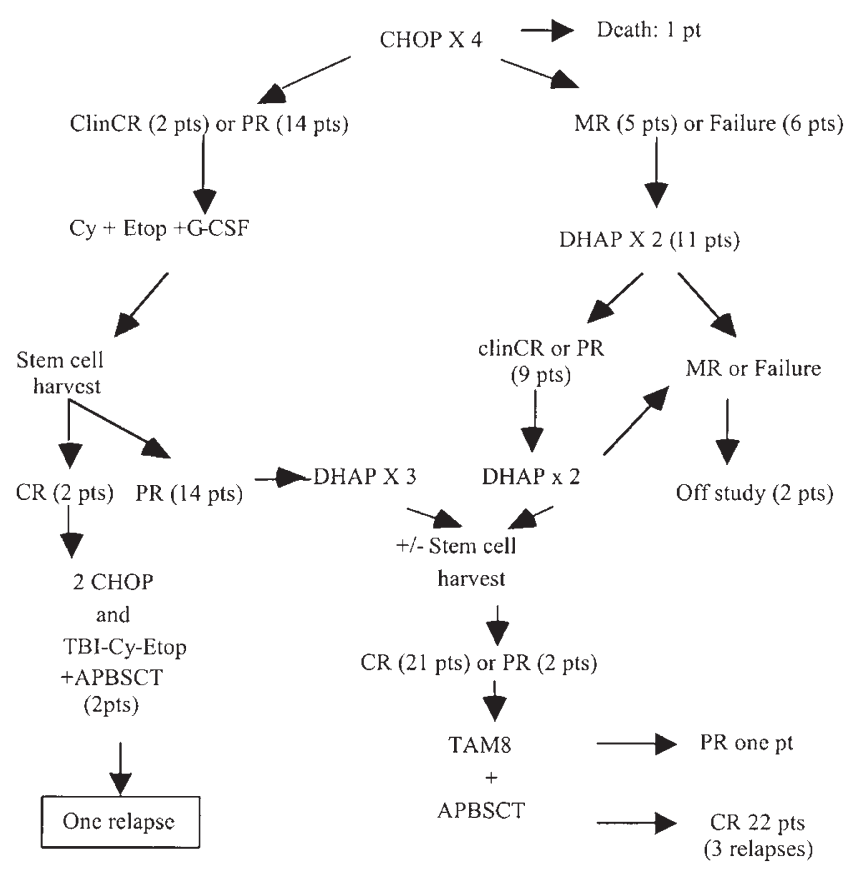

Figure 1 Protocol design and response rate after treatment. clinCR, clinical complete response; $C R$, complete response (clinCR and negative bone marrow result); $P R$, partial response; $M R$, minor response; $\mathrm{TBI}$, total body irradiation; APBSCT, autologous peripheral blood stem cell transplantation; $\mathrm{Cy}+$ Eto, cyclophosphamide + etoposide; $\mathrm{CHOP}$, cyclophosphamide $750 \mathrm{mg} / \mathrm{m}^{2}$ day 1 , adriamycin $50 \mathrm{mg} / \mathrm{m}^{2}$ day 1 , vincristine $1.4 \mathrm{mg} / \mathrm{m}^{2}$ day 1 , prednisone $40 \mathrm{mg} / \mathrm{m}^{2} /$ day days $1-5$; DHAP, cisplatin $100 \mathrm{mg} / \mathrm{m}^{2}$ day 1 , cytarabine $4 \mathrm{~g} / \mathrm{m}^{2}$ day 2, dexamethasone $40 \mathrm{mg} /$ day days $1-4$; TAM8, TBI $12 \mathrm{G}$ days $1-3$, cytarabine $4 \mathrm{~g} / \mathrm{m}^{2} /$ day days 5-6, melphalan $140 \mathrm{mg} / \mathrm{m}^{2}$ at day 8; Pt, patient.

DHAP. Autologous unpurged PBSC transplantation was scheduled within 6 weeks after the last DHAP using the TAM8 regimen (TBI 12 Gy days $1-3$, cytarabine $4 \mathrm{~g} / \mathrm{m}^{2} /$ day days $5-$ 6, melphalan $140 \mathrm{mg} / \mathrm{m}^{2}$ day 8 ). TBI was performed over 3 days as twice daily fractions at a dose of 200 cGy treatment for a total dose of 1200 cGy (800 cGy on the lungs). Patients who did not exhibit PR or CR after DHAP were not eligible for intensification by TAM8.

A novel complete clinical evaluation was planned for each patient just before and 3 months after intensification regimen and then every 4 months.

\section{Response and toxicity}

Complete clinical response (clinCR) was defined as the disappearance of all clinical and morphological evidence of lymphoma. Complete response (CR) was defined as clinCR plus normal bone marrow biopsy. PR was defined as a reduction of more than $50 \%$ of the initial tumor mass, MR as a reduction of less than $50 \%$ of the initial tumor mass and/or of the lymphocytosis. Failure or progressive disease was defined as stability or as any increase greater than $25 \%$ of the tumor mass, respectively. Toxicity was evaluated according to World Health Organization (WHO) criteria.

\section{Statistical method}

The Stuart-Maxwell test was used to evaluate the response after $\mathrm{CHOP}$ and after $\mathrm{CHOP}$ followed by the DHAP regimen 
( $\chi^{2}$ test). Event-free survival (EFS) was calculated from entry into the protocol until death, progressive disease or last contact. Survival was calculated from the date of study entry to the date of death whatever the cause. Overall survival curves were plotted using the Kaplan-Meier method. ${ }^{19}$ Statistical analysis was performed on the S. Plus 4.5 software.

\section{Results}

\section{Patients}

Between March 1995 and June 1999, 17 consecutive patients from Necker Hospital and 11 patients from other French centers were registered. Patients' characteristics are listed in Table 1. All patients had stage III/IV disease. Twenty-five patients had bone marrow involvement and malignant lymphoid cells were observed in the blood of 14 patients. Eleven out of 23 patients investigated had a gastrointestinal involvement. LDH value was found above normal count in 12 patients. Elevated $\beta 2$-microglobulin level was observed in 14 patients. PS did not exceed 1 for all patients (WHO classification). Diagnosis criteria for mantle cell lymphoma are listed for each patient in Table 2.

\section{Response}

In Figure 1 the response rates are summarized after chemotherapy and high-dose therapy. Twenty-five patients received the four planned cycles of CHOP regimen. Three patients did not complete this regimen. One patient died of septic complication after the third course (patient 2), one patient presented recurrent cyclophosphamide severe allergic pneumopathy (patient 4) and one was in clinical progression after one course (patient 16). Two clinCR (7\%) were obtained, 14 patients (50\%) and five patients (18\%) achieved PR and MR, respectively (Table 3 ). Failure or progression was noted for six $(21 \%)$. One patient who died from septic complication could not be evaluated (4\%). The 14 partial responding patients

Table 1 Initial clinical features of patients

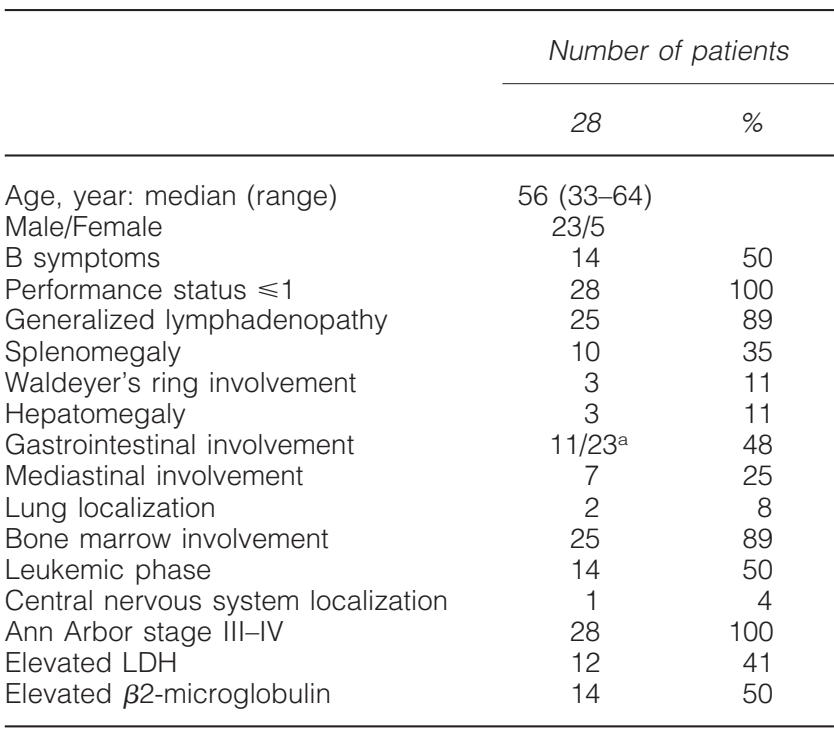

anly 23 patients underwent gastrointestinal endoscopy. The remaining five had no gastrointestinal signs.

were treated by one course of Cy-Eto for PBSC collection and

did not exhibit further significant improvement as assessed by a new evaluation including bone marrow biopsy. Thus, only two patients reached CR after CHOP plus Cy-Eto (patients 20 and 21). The other twenty-five surviving patients received the DHAP regimen.

After DHAP, CR and PR (the two PR being $>75 \%$ ) were observed for $21(84 \%)$ and two (8\%) patients, respectively (Table 3). Two progressions occurred (patients 10 and 22).

The two patients in CR after CHOP and Cy-Eto received the intensification therapy with high-dose Cy-Eto and APBSCT. The twenty-three patients who responded and survived after DHAP underwent therapeutic intensification by TAM8 regimen. Among the two partial responders after DHAP, one patient in PR achieved CR following TAM8 plus APBSCT (patient 8). Our strategy induced a total response rate (CR + $\mathrm{PR})$ of $0.78 \%$ (95\% confidence interval $(\mathrm{Cl}), 0.59$ to 92$)$.

\section{Stem cell mobilization}

Autologous PBSC mobilization was attempted after Cy-Eto plus G-CSF for the 16 patients with partial or complete response after the $\mathrm{CHOP}$ regimen. Others had stem cell mobilization following DHAP plus G-CSF. All patients achieved an adequate hematopoietic progenitor collection with a median of $6.8 \times 10^{6} \mathrm{CD}^{+} 4^{+}$cells $/ \mathrm{kg}$ harvested (range, 2.2-19).

\section{Event-free survival and survival}

Median follow-up from diagnosis and initial therapy is 47.6 months (range, 14-70 months). Median survival has still not been reached. One of the two patients treated by TBI plus cyclophosphamide-etoposide relapsed at 12 months (patient 20). Six patients relapsed after CR following the TAM8 regimen (patient 6 (48 months), patient 7 (63 months), patient 13 (40 months), patient 14 (48 months) and patient 16 (54 months), patient 19 (23 months). Median follow-up time from CR is 33 months (range, 9-61 months). The EFS and survival are shown in Figures 2 and 3, respectively. The EFS rate at 3 years is $83.3 \%(95 \% \mathrm{Cl}, 69.2$ to 100$)$. The overall survival rate at 3 years is $90.4 \%(95 \% \mathrm{Cl}, 78.2$ to 100$)$. Due to the low number of events, no prognostic factors are evaluable.

\section{Toxicity}

WHO grades 3 and 4 for granulocytopenia and/or thrombocytopenia were observed in eight (29\%) and 25 (100\%) patients after $\mathrm{CHOP}$ and DHAP regimen, respectively. One patient died from pneumococcus septic complication during CHOPinduced neutropenia but no treatment-related death was observed after DHAP and APBSCT. Severe mucositis (grade $>2, \mathrm{WHO}$ ) occurred in all patients following the TAM8 regimen. Five patients presented resolutive but severe idiopathic interstitial pulmonary syndrome (grade 3 or $4, \mathrm{WHO}$ ) after the TAM8 regimen before white blood cell recovery, and were treated with large spectrum antibiotic and symptomatic treatment. One patient needed transient artificial ventilation. Diffuse pulmonary infiltrates were present on scan and bacteriological results from bronchoalveolar aspiration were negative in all cases. All patients recovered without pulmonary sequellae. Extensive or recurrent VZV infection was observed in seven patients after hematopoietic recovery fol- 
Table 2 Histologic, immunologic, cytogenetic and molecular data

\begin{tabular}{|c|c|c|c|c|c|c|c|}
\hline \multirow[t]{2}{*}{ Patient No. } & \multirow{2}{*}{$\begin{array}{l}\text { Histologic } \\
\text { form }\end{array}$} & \multicolumn{3}{|c|}{ Immunophenotype } & \multirow[t]{2}{*}{ Cytogenetic } & \multirow{2}{*}{$\begin{array}{c}\text { Overexpression } \\
\text { of cyclin D1 } \\
\text { (slot blot analysis) }\end{array}$} & \multirow{2}{*}{$\begin{array}{c}\mathrm{BCl1-lgH} \\
\text { rearrangement } \\
\text { (PCR analysis) }\end{array}$} \\
\hline & & CD5 & CD23 & FMC7 & & & \\
\hline 1 & Nodular & + & NP & NP & NP & + & - \\
\hline 2 & Diffuse & + & - & + & NP & - & + \\
\hline 3 & Diffuse & + & NP & NP & NP & + & + \\
\hline 4 & Diffuse & + & - & + & $\mathrm{t}(11 ; 14)$ & + & - \\
\hline 5 & Nodular & + & - & - & $\mathrm{N}$ & - & - \\
\hline 6 & Diffuse & + & - & + & $\mathrm{t}(11 ; 14)$ & + & - \\
\hline 7 & Diffuse blastic & + & - & + & $\mathrm{t}(11 ; 14)$ & + & - \\
\hline 8 & Nodular & + & - & + & trisomy 12 & - & - \\
\hline 9 & Nodular & + & - & + & $\begin{array}{c}\mathrm{t}(11 ; 14) \\
\text { trisomy } 3\end{array}$ & + & - \\
\hline 10 & Diffuse & + & - & NP & $N$ & + & - \\
\hline 11 & Diffuse & + & + & + & $\begin{array}{c}\mathrm{t}(11 ; 14) \\
\text { trisomy } 3\end{array}$ & NP & NP \\
\hline 12 & Nodular & + & - & + & N & NP & NP \\
\hline 13 & Diffuse & + & NP & NP & $\begin{array}{l}t(11 ; 14) \\
t(10 ; 13)\end{array}$ & NP & NP \\
\hline 14 & Diffuse & + & - & + & NP & + & + \\
\hline 15 & Diffuse & + & - & NP & NP & + & + \\
\hline 16 & Nodular & + & - & + & $\mathrm{t}(11 ; 14)$ & + & + \\
\hline 17 & Nodular & + & - & + & $\begin{array}{l}\mathrm{t}(11 ; 14) \\
\text { del }(6 q)\end{array}$ & + & + \\
\hline 18 & Nodular & + & - & + & $\mathrm{t}(11 ; 14)$ & + & + \\
\hline 19 & Diffuse & + & - & + & $\begin{array}{l}t(11 ; 14) \\
\text { del 3p21 }\end{array}$ & + & + \\
\hline 20 & Diffuse & + & - & + & del(13) & NP & NP \\
\hline 21 & Diffuse & + & - & NP & $N$ & NP & NP \\
\hline 22 & Diffuse & + & - & NP & $\mathrm{t}(11 ; 14)$ & + & - \\
\hline 23 & Diffuse & + & - & + & $\begin{array}{l}t(11 ; 14) \\
\text { del (6) }\end{array}$ & + & + \\
\hline 24 & Diffuse & + & - & + & $\mathrm{t}(11 ; 14)$ & + & + \\
\hline 25 & Nodular & + & - & NP & NP & + & + \\
\hline 26 & Nodular & + & - & + & $N$ & - & + \\
\hline 27 & Diffuse & + & - & NP & $\mathrm{t}(11 ; 14)$ & + & - \\
\hline 28 & Diffuse & + & - & + & $t(11 ; 14)$ & + & + \\
\hline
\end{tabular}

NP, not performed; N, normal.

lowing TAM8 and APBSCT. Despite a sufficient CD34 ${ }^{+}$cell count transfused after TAM8 regimen, one patient did not reach a sufficient hematologic recovery following high-dose therapy and APBSCT and required regular red blood cell transfusion for 1 year.

\section{Discussion}

MCL is a B cell lymphoproliferation well individualized in the recent lymphoma classifications, characterized by its refractoriness to therapeutic strategies with a very poor prognosis. ${ }^{3,8-11} \mathrm{MCL}$ exhibits the shortest median time to progression and the shortest median survival of all small B cell lymphoma subtypes. ${ }^{8,10}$ In most studies, whatever the initial therapy, patients present partial regression of the lymphoma for 6 to 18 months and then progress and die with a median overall survival of 3 to 4 years. ${ }^{9,21}$ We report here, that DHAP regimen is very efficient in inducing $C R$ in patients who did not fully respond to $\mathrm{CHOP}$ regimen. Furthermore, although median follow-up is still short, intensification with the TAM8 regimen followed by APBSCT seems to prolong EFS and survival from historical studies ${ }^{3,8-11}$ and may induce molecular remission at the $10^{-4}$ sensitivity.

All patients fulfilled the histological, immunophenotypic, molecular or cytogenetic criteria of aggressive MCL except patients 5 and 8 who exhibited negative Bcl1-lgH rearrangement and no dectectable overexpression of cyclin D1 from the sample studied. All patients presented severe and advanced disease and our series confirms the classic clinical presentation of aggressive MCL with a median age of 55 years (46$62)$, male preponderance $(90 \%)$, and frequent extranodal disease $(100 \%)$ with bone marrow and/or gastrointestinal involvement. Moreover, half of the patients had an increased $\beta 2$-microglobulin level, which has been reported as a poor prognosis factor in MCL. ${ }^{22}$ Patients with mantle zone pattern, because of their relatively good prognosis (CR rate of $75 \%$ and 3 -year survival rate of $100 \%$ ) were not included in this trial. ${ }^{18}$ The $t(11 ; 14)$ and overexpression of cylin D1 were found in $56 \%$ and $81 \%$ of investigated patients, respectively. Therefore our group of patients was comparable with other previous published studies. ${ }^{14,22}$

After the first line therapy with the CHOP regimen, two CR were obtained and $50 \%$ of our patients reached a PR. This finding is in agreement with some reports ${ }^{22}$ but much lower than others. ${ }^{1,21}$ Majlis et a/18 reported in newly diagnosed patients a CR rate of $20 \%$ with a CHOP-like regimen. Furthermore, one previous study which evaluated increased $\mathrm{CHOP}$ like regimen in 18 patients showed only one CR while other patients presented PR or were refractory. ${ }^{14}$ In contrast, other retrospective studies using doxorubucin-containing regimens have shown high response rates ranging from $60 \%$ to $83 \% .^{1,21}$ 
Table 3 Clinical response after therapy and follow-up

\begin{tabular}{llccc}
\hline $\begin{array}{l}\text { Patient } \\
\text { No. }\end{array}$ & No. of & Response & Response \\
after CHOP & TAM8 DHAP & Response & $\begin{array}{c}\text { Follow-up from } \\
\text { after graft diagnosis and status } \\
\text { (months) }\end{array}$
\end{tabular}

\begin{tabular}{|c|c|c|c|c|c|c|c|}
\hline 1 & 4 & MR & PR & yes & PR & Evolutive on 39th month & Alive CR2 48th month \\
\hline 2 & 3 & $\mathrm{NE}^{\mathrm{a}}$ & - & - & - & - & Dead \\
\hline 3 & 4 & PR & CR & yes & CR & 47 & CR \\
\hline 4 & $2^{b}$ & PR & CR & yes & CR & 44 & CR \\
\hline 5 & 4 & failure & CR & yes & CR & 44 & CR \\
\hline 6 & 4 & PR & CR & yes & CR & Relapse 48th month & CR \\
\hline 7 & 4 & PR & CR & yes & CR & Relapse 63rd month & Dead 70th month \\
\hline 8 & 4 & failure & PR & yes & CR & 45 & CR \\
\hline 9 & 4 & PR & CR & yes & CR & 67 & CR \\
\hline 10 & 4 & PR & progression & no & - & Progression 13th month & Dead 21st month \\
\hline 11 & 4 & MR & $\mathrm{CR}$ & yes & CR & 30 & CR \\
\hline 12 & 4 & PR & CR & yes & CR & 30 & CR \\
\hline 13 & 4 & failure & CR & yes & CR & Relapse 40th month & Alive and PR2 42nd month \\
\hline 14 & 4 & MR & CR & yes & CR & Relapse 48th month & Alive and PR2 58th month \\
\hline 15 & 4 & failure & CR & yes & CR & 57 & CR \\
\hline 16 & 1 & progression & CR & yes & CR & Relapse 54th month & Alive 63rd month \\
\hline 17 & 4 & progression & CR & yes & CR & 45 & CR \\
\hline 18 & 4 & PR & CR & yes & CR & 60 & CR \\
\hline 19 & 4 & PR & CR & yes & CR & Relapse 23rd month & Alive and PR2 26th month \\
\hline 20 & 4 & CR & - & - & CR & Relapse 12th month & Alive and PR2 18th month \\
\hline 21 & 4 & CR & - & - & CR & 21 & CR \\
\hline 22 & 4 & PR & Progression & no & - & Progression 14th month & Alive in MR 18th month \\
\hline 23 & 4 & PR & CR & yes & CR & 22 & $\mathrm{CR}$ \\
\hline 24 & 4 & PR & CR & yes & CR & 20 & CR \\
\hline 25 & 4 & PR & CR & yes & CR & 18 & CR \\
\hline 26 & 4 & PR & CR & yes & CR & 18 & CR \\
\hline 27 & 4 & MR & CR & yes & CR & 15 & CR \\
\hline 28 & 4 & MR & CR & yes & CR & 14 & CR \\
\hline
\end{tabular}

CR, complete response, PR, partial response, MR, minor response, NE, not evaluable.

apatient died of septic complications.

${ }^{b}$ Recurrent cyclophosphamide allergic pneumopathy.

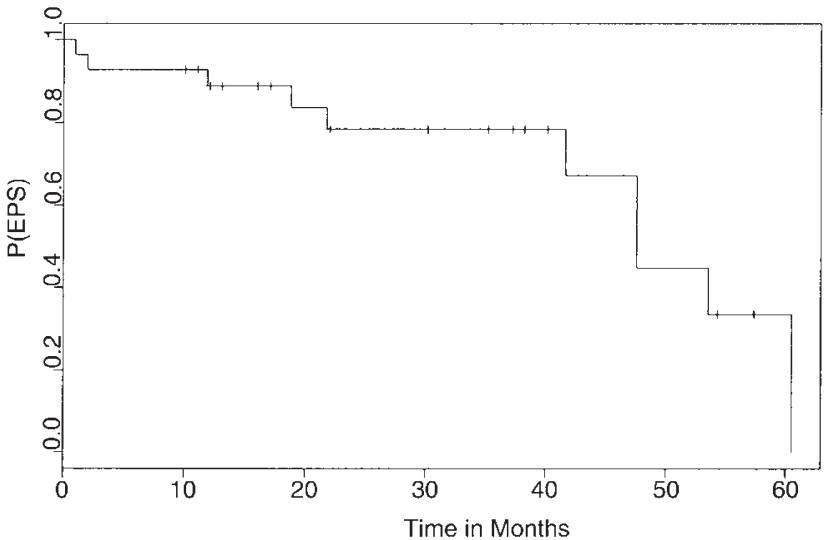

Figure 2 Event-free survival (Kaplan-Meier).

Likewise, the continuous infusion of anthracycline using the classical VAD regimen associated or not with chlorambucil showed in MCL patients a $43 \%$ rate of CR. ${ }^{23}$ However, response criteria in all these studies did not include bone marrow and gastrointestinal examination. Taken together, these studies suggest that for a large proportion of patients with $\mathrm{MCL}, \mathrm{CHOP}$ or CHOP-like regimens failed to obtain a minimal tumor burden before stem cell collection and intensive therapy plus APBSCT.

In order to increase the response rate before APBSCT, we have tested a second line therapy with high-dose Ara-c regi-

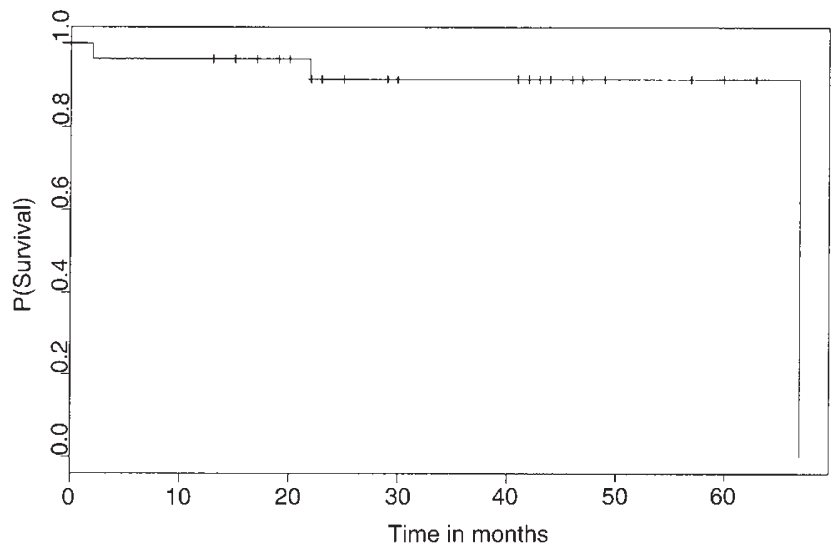

Figure 3 Overall survival (Kaplan-Meier)

men (DHAP). We have chosen DHAP because high-dose Ara$C$ is an effective therapy in obtaining complete response in patients in relapse of aggressive NHL. ${ }^{23-25}$ Furthermore, this drug allows intensification with APBSCT and may induce cure of aggressive relapsing NHL. In our series this regimen produced a high rate of CR (84\%) and good PR (8\%) after failure of $\mathrm{CHOP}$ without life-threatening toxicity. However, due to the particular course of MCL and the design of our protocol, the impact of DHAP cannot be dissociated from the previous therapy by $\mathrm{CHOP}$ and we could not totally exclude that four more courses of CHOP could have induced CR in a subgroup 
of good partial responder patients. Therefore, we cannot conclude that DHAP alone used as first line therapy would induce similar responses as those obtained after the sequence $\mathrm{CHOP}_{-}$ DHAP. This question might be solved by setting-up a crossrandomized study comparing $\mathrm{CHOP}$ and DHAP followed by DHAP or CHOP, respectively, in case of no response after the first line therapy. Our data are in agreement with the results of a recently published study using the hyper-CVAD/MTX-Ara$\mathrm{c}$ regimen that contains high-dose Ara-c. Indeed, in this study, the authors reported a high response rate of $93.5 \%$ (CR 38\%, PR 55\%) for patients with MCL previously treated (or not). ${ }^{26}$ However, the response rate for the subgroup of previously untreated patients was not given and some patients (some of whom have been previously treated with chemotherapy) failed to obtain enough PBSC yield for autografting. It is noteworthy that in our study two courses of DHAP after four $\mathrm{CHOP}$ cycles did not compromise stem cell harvesting, since no patient failed to have adequate peripheral stem cell collection.

Earlier reports suggested that a TBI conditioning regimen before PBSC transplantation improved the prognosis of $\mathrm{MCL}^{14}$ compared to other procedures. The high response rate that we observed with DHAP suggests that high-dose Ara-c may be included in the conditioning regimen. Thus, we have chosen the TAM8 regimen, which contains both Ara-C and TBI, and which is a feasible conditioning regimen as previously shown in the treatment of acute lymphoblastic leukemia. ${ }^{15}$

All our partial or complete responder patients after DHAP undertook intensification by TAM8 plus APBSCT. One out of two in PR before TAM8 achieved CR, emphasizing the value of the conditioning regimen. No conditioning transplantrelated mortality was registered. However, the morbidity was high with occurrence of many recurrent and/or extensive herpes-zoster reactivations. Thus, a prophylactic therapy with acyclovir should be recommended following TAM8 regimen. Moreover, five patients presented severe but resolutive interstitial pulmonary syndrome without identified etiology. Thus, such therapeutic strategy needs accurate pulmonary evaluation before high-dose therapy. One patient exhibited late platelet and hemoglobin recovery and was transfusion dependent for 1 year after autograft despite an adequate number of CD34 ${ }^{+}$cells being transfused.

After a median follow-up of 27 months after CR (and 37 months after diagnosis and initial therapy), $83 \%$ of autografted patients remain in CR. These results strongly suggest that our treatment strategy may improve EFS when compared with historical studies. ${ }^{8-13}$ These results are encouraging because long-term disease-free survival is uncommon for patients with MCL, especially for those with advanced disease. ${ }^{27}$ However, relapses occurred in patients with longer follow-up, which may suggest that the curve is not yet achieved. Recent studies have reported that anti-CD20 monoclonal antibodies may induce significant response rates in MCL relapsing patients. ${ }^{28}$ In future protocols, our results may be improved by using a combination of CHOP/DHAP and anti-CD20 as suggested by Romaguera et $a^{29}$ with the combination of HyperCVAD and anti-CD20.

In conclusion, stimulating results were obtained after $\mathrm{CHOP}$ followed by salvage therapy with DHAP and further APBSCT after TAM8 regimen which argues for the use of a high-dose Ara-c regimen in the induction therapy for patients with MCL. A further step with consolidation therapy by TBI and highdose Ara-c sustained by APBSCT seems promising to improve the outcome of MCL patients. Longer follow-up is, however, warranted to assess the impact of our strategy on survival.

\section{References}

1 Zucca E, Stein H, Coiffier B. European Lymphoma Task Force (ELTF). Report of the workshop on mantle cell lymphoma (MCL). Ann Oncol 1994; 5: 507-511.

2 Harris NL, Jaffe ES, Stein H, Banks PM, Chan JK, Cleary ML, Delsol MG, De Wolf-Peeters, Falini B, Gatter KC. A revised EuropeanAmerican classification of lymphoid neoplam: a proposal from the International Lymphoma Study Group. Blood 1994; 84 (Suppl. 6): 1361-1392.

3 Weisenburger DD, Armitage JO. Mantle cell lymphoma - an entity comes of age. Blood 1996; 87: 4483-4494.

4 Zucca E, Soldati G, Schlegelbeger B, Booth MJ, Weber-Matthiesen K, Cavalli F, Cotter FE. Detection of chromosome 11 alterations in blood and bone marrow by interphase cytogenetics in mantle cell lymphoma. Br J Haematol 1995; 89: 665-668.

5 Uchimaru K, Taniguchi T, Yoshikawa M, Asano S, Arnold A, Fujita $\mathrm{T}$, Motokura T. Detection of cyclin D1 overexpression by a simple competitive reverse transcription-polymerase chain reaction assay in $\mathrm{t}(11 ; 14)$-bearing B-cell malignancies and/or mantle cell lymphoma. Blood 1997; 89: 965-974.

6 Levy V, Ugo V, Delmer A, Tang R, Ramond S, Perrot JY, Vrhovac $R$, Marie JP, Zittoun R, Ajchenbaum-Cymbalista F. Cyclin D1 overexpression allows identification of an aggressive subset of leukemic lymphoproliferative disorder. Leukemia 1999; 13: 13431351 .

7 Mauvieux L, Canioni D, Hermine O, Valensi F, Radford-Weiss, Azagury M, Magen M, Flandrin G, Brousse N, Varet B, Maclntyre EA. Quantitative RNA slot-blot analysis of CCND1/cyclin D1 expression in suspected mantle cell lymphoma. Leukemia 1998; 12: 78-85.

8 Pittaluga S, Bijnens L, Teodorovic I, Hagenbeek A, Meerwaldt JH, Somers R, Thomas J, Noordijk EM, De Wolf-Peeters C. Clinical analysis of 670 cases in two trials of the European Organization for the Research and Treatment of Cancer Lymphoma Cooperative group subtype according to the revised European-American classification of lymphoid neoplasms: a comparison with the Working Formulation. Blood 1996; 87: 4358-4367.

9 Bosch F, Lopez-Guillermo A, Campo E, Ribera JM, Conde E, Piris MA, Vallespi T, Woessner S, Montserrat E. Mantle cell lymphoma: presenting features, response to therapy, and prognosis factors. Cancer 1998; 82: 567-575.

10 Vandenberghe E, De Wolf-Peeters C, Vaughan Hudson G, Vaughan Hudson B, Pittaluga S, Anderson L, Linch DC. The clinical outcome of mantle cell lymphoma initially treated with nonintensive therapy by the British National Lymphoma Investigation Group. Br J Haematol 1997; 99: 842-847.

11 Segal GH, Masih AS, Fox AC, Jorgensen T, Scott M, Braylan RC. CD5-expressing B cell non Hodgkin's lymphomas with bcl-1 gene rearrangement have a relatively homogeneous immunophenotype and are associated with an overall poor prognosis. Blood 1995; 85: 1570-1579.

12 Fisher RI, Dahlberg S, Nathwani BN, Banks PM, Miller TP, Grogan TM. A clinical analysis of the two indolent lymphoma entities: mantle cell lymphoma and marginal zone lymphoma (including the mucosa-associated lymphoid tissue and monocytoid B-cell subcategories): a Southwest Oncology Group Study. Blood 1995; 85: 1075-1082.

13 Meusers P, Engelhard M, Bartels H, Binder T, Fulle HH, Gorg K, Gunzer U, Havemann K, Kayser W, Konig E. Multicentre randomized therapeutic trial for advanced centrocytic lymphoma: anthracycline does not improve the prognosis. Hematol Oncol 1989; 7: 365-380.

14 Milpied N, Gaillard F, Moreau P, Mahe B, Souchet J, Rapp MJ, Bulabois CE, Morineau N, Harousseau JL. High-dose therapy with stem cell transplantation for mantle cell lymphoma: results and prognostic factors, a single center experience. Bone Marrow Transplant 1998; 22: 645-650.

15 Cahn JY, Bordigoni P, Souillet G, Pico JL, Plouvier E, Reiffers J, Benz-Lemoine E, Bergerat JP, Lutz P, Colombat P. The TAM regimen, prior to allogenic and autologous bone marrow transplantation for high-risk acute lymphoblastic leukemias: a cooperative study of 62 patients. Blood 1991; 7: 1-4.

16 Carbone P-P, Kaplan H, Musshoff K, Smithers DW, Tubiana M. Report on the committee on Hodgkin's disease staging classification. Cancer Res 1971; 31: 1860-1861. 
17 Geissmann F, Ruskone-Fourmestraux A, Hermine O, Bourquelot P, Belanger C, Audouin J, Delmer A, Macintyre EA, Varet B, Brouuse N. Homing receptor alpha4beta7 integrin expression predicts digestive tract involvement in mantle cell lymphoma. Am J Pathol 1998; 153: 1701-1705.

18 Majlis A, Pugh W-C, Rodriguez M-A, Benedict WF, Cabanillas F. Mantle cell lymphoma: correlation of clinical outcome and biologic features with three histologic variants. J Clin Oncol 1997; 15: 1664-1671.

19 Kaplan E-L, Meier P. Nonparametric estimation from incomplete observations. J Am Stat Assoc 1958; 53: 457-458.

20 Shipp M-A. A predictive model for aggressive non-Hodgkin's lymphoma. The International non-Hodgkin's lymphoma prognostic factors. N Engl J Med 1993; 329: 987-994.

21 Teodorovic I, Pittaluga S, KLuin-Nelemans J-C, Meerwaldt JH, Hagenbeek A, Van Glabbeke M, Somers R, Bijnens L, Noordijk EM, Peeters CD. Efficacy of four different regimens in 64 mantlecell lymphoma cases: clinicopathologic comparison with 498 other non-Hodgkin's lymphoma subtypes. J Clin Oncol 1995; 13 2819-2826.

22 Samaha H, Dumontet C, Ketterer N Moullet I, Thieblemont C, Bouafia F, Callet-Bauchu E, Felman P, Berger F, Salles G, Coiffier B. Mantle cell lymphoma: a retrospective study of 121 cases. Leukemia 1998; 12: 1281-1287.

23 Gressin R, Legouffe E, Leroux D, Jacob MC, Swiercz P, Peoch M, Capdevilla V, Rossi JF, Thyss A, Sotto JJ. Treatment of mantle celllymphoma with the $\mathrm{VAD}+/$ - chlorambucil regimen with or without subsequent high dose therapy and peripheral blood stem cell transplantation. Ann Oncol 1997; 8: (Suppl. 1): 103-106.

24 Press OW, Livingston R, Mortimer J, Collin C, Appelbaum F. Treatment of relapsed non-Hodgkin's lymphomas with dexamethasone,

high-dose cytarabine and cisplatin before marrow transplantation. J Clin Oncol 1991; 7: 1587-1592.

25 Velasquez WS, Cabanillas F, Salvador P, McLaughlin P, Fridrik M, Tucker S, Jagannath S, Hagemeister FB, Redman JR, Swan F. Effective therapy for lymphoma with cisplatin in combination with high-dose Ara-C and dexamethasone (DHAP). Blood 1988; 71: 117-122.

26 Khouri I, Romaguera J, Kantarjian H, Palmer JL, Pugh WC, Korbling M, Hagemeister F, Samuels B, Rodriguez A, Giralt S, Younes A, Pzrepiorka D, Claxton D, Cabanillas F, Champlin R. HyperCVAD and high-dose methotrexate/cytarabine followed by stem cell transplantation: an active regimen for aggressive mantle-cell lymphoma. J Clin Oncol 1998; 16: 3803-3809.

27 Corradini P, Astolfi M, Cherasco C, Ladetto M, Voena C, Carraciolo D, Pileri A. Molecular monitoring of minimal residual disease in follicular and mantle cell non-Hodgkin's lymphomas treated with high-dose chemotherapy and peripheral blood progenitor cell autografting. Blood 1997; 89: 724-731.

28 Foran J, Cunningham D, Coiffier B, Solal-Celigny P, Reyes F, Ghielmini M, Johnson PW, Gisselbrecht C, Bradburn M, Matthews J, Lister T. Treatment of mantle cell lymphoma with Rituximab (chimeric anti-CD20 monoclonal antibody) for patients with newly diagnosed mantle cell lymphoma and previously treated mantle cell lymphoma, immunocytoma and small lymphocytic lymphoma. J Clin Oncol 2000; 18: 317-324.

29 Romaguera J, Dang N, Hagemeister F, McLaughlin P, Pro B, Rodriguez M, Samaniego P, Sarris A, Younes A, Medeiros J, Steinbach G, Cabanillas F. Preliminary report of Rituximab with intensive chemotherapy for untreated aggressive mantle cell lymphoma. Blood 2000; 96: 733 (Abstr. 3170). 